\title{
AN OPEN MAPPING THEOREM FOR PRO-LIE GROUPS
}

\author{
KARL H. HOFMANN and SIDNEY A. MORRIS $\cong$
}

(Received 4 July 2005; revised 10 April 2006)

Communicated by $\mathrm{G}$. Willis

\begin{abstract}
A pro-Lie group is a projective limit of finite dimensional Lie groups. It is proved that a surjective continuous group homomorphism between connected pro-Lie groups is open. In fact this remains true for almost connected pro-Lie groups where a topological group is called almost connected if the factor group modulo the identity component is compact. As consequences we get a Closed Graph Theorem and the validity of the Second Isomorphism Theorem for pro-Lie groups in the almost connected context.
\end{abstract}

2000 Mathematics subject classification: primary 22A05, 22E65; secondary 46A30.

\section{Introduction}

The identity morphism $f: \mathbb{R}_{d} \rightarrow \mathbb{R}$ from the discrete group of real numbers to the group of real numbers with its natural topology illustrates that a surjective morphism between locally compact metric groups (indeed Lie groups) may fail to be open. It is accepted parlance to apply the title Open Mapping Theorem to any statement that asserts that a surjective morphism of topological groups is automatically open provided that certain additional hypotheses are satisfied. Open surjective morphisms are equivalent to quotient morphisms. So whenever an Open Mapping Theorem applies to a surjective morphism of topological groups we know that it is (equivalent to) a quotient morphism: a very important piece of information. There is a considerable body of literature, notably in functional analysis, on Open Mapping Theorems and their corollaries. The best known examples in topological group theory are as follows.

(C) 2007 Australian Mathematical Society 1446-7887/07 \$A2.00+0.00 
THEOREM A. (The Open Mapping Theorem for Locally Compact Groups) A surjective morphism of locally compact groups is open if the domain group is a countable union of compact sets. (See [3, page 42, Theorem 5.29].)

A topological space is called a Polish space if it is completely metrizable and has a countable basis for its topology.

THEOREM B. (The Open Mapping Theorem for Polish Groups) A surjective morphism between Polish groups is open. (See, for instance, [12].)

In functional analysis, the following theorem is exemplary and found in almost all text books on functional analysis; it is not subsumed under Theorem $B$ because it does not require separability.

THEOREM C. (The Open Mapping Theorem in Functional Analysis) A surjective linear map between first countable complete topological vector spaces (Frechet spaces) is open.

All of these theorems rest on the fact that the underlying space of the range group has the property that whenever it is written as the union of a countable family of closed subspaces, one family member has to have a nonempy interior. This is true for all Baire spaces. Every locally cumpact and every locally completely metrizable space is a Baire space. (See, for instance, [2, Chapter IX, Section 5, number 3, Theorem 1].)

A surjective morphism $f: G \rightarrow H$ of connected real Lie groups is open by Theorem B if $G$ is separable. This covers all finite dimensional connected Lie groups, a case that is also covered by Theorem A. Recall from the standard source for the foundation of Lie groups [1, Chapter III, Section 1, Number 1, Définition 1], that Lie groups may perfectly well be defined on infinite dimensional manifolds modelled on completely normable topological vector spaces. In order to make the point that Open Mapping Theorems are problematic even in the Lie group context, we mention that the Open Mapping Theorem between separable Lie groups breaks down if $G$ fails to be separable and hence finite dimensional (see, for instance, [5, Example following Proposition 5.52], where the example id: $\mathbb{R}_{d} \rightarrow \mathbb{R}$ is embedded into an infinite dimensional connected abelian Lie group).

However, all Lie groups we consider in this paper are assumed to be finite dimensional real Lie groups and all topological groups are assumed to be Hausdorff.

Recall that a filter on a topological group $G$ is called a Cauchy filter if for every identity neighborhood $U$ there is a set $F$ in the filter such that $F^{-1} F \subseteq U$. The group $G$ is called complete if every Cauchy filter converges.

PROPOSITION 1.1. For a topological group $G$ the following statements are equivalent. 
(i) There is a projective system $\left\{f_{j k}: G_{k} \rightarrow G_{j} \mid j \leq k,(j, k) \in J \times J\right\}$ of finite dimensional Lie groups such that $G=\lim _{j \in J} G_{j}$.

(ii) $G$ is isomorphic as a topological group to a closed subgroup of a product $\prod_{j \in J} G_{j}$ of finite dimensional Lie groups.

(iii) $G$ is complete and each identity neighborhood of $G$ contains a normal subgroup $N$ such that $G / N$ is a finite dimensional Lie group.

(See [6], or [9, Chapter 3]. The proof of the implication 'anything else implies.(iii)' is nontrivial.)

DEFINITION 1.2. A topological group satisfying the equivalent conditions of Proposition 1.1 is called a pro-Lie group. If every identity neighborhood in $G$ contains a normal subgroup $N$ such that $G / N$ is a Lie group then $G$ is called a proto-Lie group.

Accordingly, every pro-Lie group is a proto-Lie group and a proto-Lie group is a pro-Lie group if and only if it is complete. To see this, note that for every proto-Lie group $G$, the set $\mathscr{N}(G)$ of closed normal subgroups of $G$ such that $G / N$ is a Lie group is a filter basis such that there is a natural embedding morphism $\gamma_{G}: G \rightarrow G_{\mathcal{N}(G)} \stackrel{\text { def }}{=} \lim _{N \in \mathscr{N}(G)} G / N$ with dense image, and $G_{\mathscr{N}(G)}$ is a pro-Lie group and is the completion of $G$ ([9, Theorem 4.1]). We shall also write the completion of $G$ as $\bar{G}$, notably when we consider $G$ as a dense subgroup of its completion. Every closed subgroup of a pro-Lie group is a pro-Lie group ([6, Theorem 4.8]). A Lie group is a proto-Lie group if and only if it is finite dimensional. If $J$ is any set, the power $\mathbb{R}^{J}$ is a pro-Lie group. If $J$ is infinite, then this group fails to be locally compact, and if $J$ is uncountable, $\mathbb{B}^{J}$ fails to be metric. All locally compact abelian groups are pro-Lie groups. A topological group $G$ is called almost connected, if the factor group $G / G_{0}$, where $G_{0}$ is the identity component of $G$, is compact. All almost connected locally compact groups are pro-Lie groups $([15,16]$, see also [13, page 175]). There is a pro-Lie group topology on the free abelian group $\mathbb{Z}^{(\mathbb{N})}$ on countably many generators, making it into a nondiscrete pro-Lie group $F([7,10]$ and $[9$, Chapter 5]). The identity morphism $\left(\mathbb{Z}^{(\mathbb{N})}\right)_{d} \rightarrow F$ from the discrete free abelian group on a countable set of generators to $F$ shows that there exists a surjective morphism between countable pro-Lie groups that is not open.

It is therefore hopeless to expect any Open Mapping Theorem for pro-Lie groups to arise from Baire category arguments directly, because a countable homogeneous Baire space is necessarily discrete. In this paper we shall prove the following theorem.

THEOREM D. (The Open Mapping Theorem for Pro-Lie Groups) A surjective morphism from an almost connected pro-Lie group onto a pro-Lie group is open.

Theorem A will enter the proof at a suitable point, but in no way can Theorem $D$ be considered as a 'generalisation' of any of the Theorems A, B or C. In the next section 
we assemble some background information that we shall use in the proof proper. In subsequent sections we present the proof of Theorem $\mathrm{D}$ and in a final section we draw some consequences from it.

\section{Background information}

Mathematicians involved in considerations of limits of topological groups or topological spaces know the concept of cofinality; the essence is that a projective system that is cofinal in another produces the same limit. Readers familiar with category theory will know that cofinality may be studied on a surprisingly general level. The following version suffices for us.

Let

$$
\mathscr{P}=\left\{f_{j k}: G_{k} \rightarrow G_{j} \mid(j, k) \in J \times J, j \leq k\right\}
$$

be a projective system of topological groups, let $\Gamma$ be a directed set and let $\theta: \Gamma \rightarrow J$ be an order preserving cofinal function, that is, for each $j \in J$ there is an $\alpha \in \Gamma$ such that $j \leq \theta(\alpha)$. For $\alpha \in \Gamma$ we set $H_{\alpha}=G_{\theta(\alpha)}$, and if $\alpha \leq \beta$ we define $\phi_{\alpha \beta}: H_{\beta} \rightarrow H_{\alpha}$ by $\phi_{\alpha \beta}=f_{\theta(\alpha) \theta(\beta)}$. Then

$$
\mathscr{P}_{\theta} \stackrel{\text { def }}{=}\left\{\phi_{\alpha \beta}: H_{\beta} \rightarrow H_{\alpha} \mid(\alpha, \beta) \in \Gamma \times \Gamma, \alpha \leq \beta\right\}
$$

is straightforwardly seen to be a projective system of topological groups, which is said to be cofinal in $\mathscr{P}$. Let $\phi_{\alpha}: H=\lim _{\beta \in \Gamma} H_{\beta} \rightarrow H_{\alpha}$ be the limit maps. Notice that an order preserving surjective function $\theta: \Gamma \rightarrow J$ is always cofinal.

LEMMA 2.1. (The Cofinality Lemma) If $H=\lim _{\alpha \in \Gamma} H_{\alpha}$ is the projective limit of a projective system $\mathscr{P}_{\theta}$ that is cofinal in a projective system $\mathscr{P}$ then $H$ is the limit of $\mathscr{P}$ with appropriate limit maps $\widetilde{\phi}_{j}: H \rightarrow G_{j}$ satisfying $\widetilde{\phi}_{\theta(\alpha)}=\phi_{\alpha} . \quad$ (See $[9$, Lemma 1.21].)

Unfortunately, quotients of pro-Lie groups may fail to be pro-Lie groups, due to their potential loss of completeness. Nevertheless, quotients of pro-Lie groups remain proto-Lie groups, as we record now.

THEOREM 2.2. (The Quotient Theorem for Pro-Lie Groups) A quotient group of a proto-Lie group is a proto-Lie group and thus is isomorphic as a topological group to a dense subgroup of a pro-Lie group. If the quotient group is complete, then it is a pro-Lie group. (See [9, Theorem 4.1].) 
Pro-Lie groups have a very effective, in general 'infinite dimensional', Lie theory. In all that follows we must refer to it. A full account is given in [9], but the basics have been introduced in [4,6-8]. The gist is as follows: for any topological group $G$ there is a functorially attached topological space $\mathfrak{L}(G) \stackrel{\text { def }}{=} \operatorname{Hom}(\mathbb{R}, G)$ of all morphisms $X: \mathbb{R} \rightarrow G$ endowed with the topology of uniform convergence on compact subsets of $\mathbb{R}$ and with a scalar multiplication defined by $(s \cdot X)(r)=X(r s)$ for $X \in \mathfrak{L}(G), r, s \in \mathbb{R}$. The morphisms $X \in \mathfrak{L}(G)$ are called one parameter subgroups. A topological Lie algebra is a Lie algebra on a topological vector space with a continuous Lie bracket multiplication. For two elements $g$ and $h$ in a group, we write $\operatorname{comm}(g, h)=g h g^{-1} h^{-1}$. One shows that for any pro-Lie group $G$, the space $\mathfrak{L}(G)$ supports a topological Lie algebra structure with an addition and bracket satisfying

$$
\begin{gathered}
X+Y=\lim _{n \rightarrow \infty}\left(\left(\frac{1}{n} \cdot X\right)\left(\frac{1}{n} \cdot Y\right)\right)^{n}, \\
{[X, Y] \circ \square=\lim _{n \rightarrow \infty} \operatorname{comm}\left(\frac{1}{n} \cdot X, \frac{1}{n} \cdot Y\right)^{n^{2}},}
\end{gathered}
$$

where we denote by $\square: \mathbb{R} \rightarrow \mathbb{R}$ the function given by $\square(t)=t^{2}$. We define the exponential function of $G$ by $\exp X=X(1)$

While passing to a quotient of a pro-Lie group may lead us outside the category of pro-Lie groups, quotient morphisms are preserved by the all-important Lie algebra functor (see [8], [9, Chapter 2]). This is expressed more explicitly as follows.

THEOREM 2.3. (i) Let $f: G \rightarrow H$ be a quotient morphism of topological groups and assume that $G$ is a pro-Lie group. Then the completion $\bar{H}$ of $H$ has the same one parameter subgroups as $H$, that is, $\mathscr{L}(H)=\mathscr{L}(\bar{H})$. In particular, $\mathscr{L}(H)$ is the Lie algebra of a pro-Lie group, namely $\bar{H}$, and there is a commutative diagram showing that $H$ and $\bar{H}$ 'have the same exponential function':

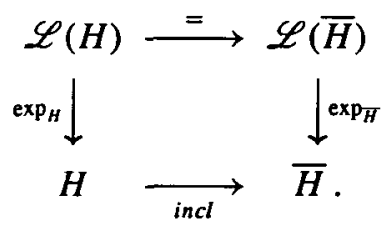

Moreover, $\mathscr{L}(f): \mathscr{L}(G) \rightarrow \mathscr{L}(H)$ is a quotient morphism of topological Lie algebras. In particular, the functor $\mathscr{L}$ is exact if

$$
N \stackrel{e}{\longrightarrow} G \stackrel{f}{\longrightarrow} H
$$

is an exact sequence of morphisms of pro-Lie groups with a morphism e that is open onto its image, then

$$
\mathscr{L}(N) \stackrel{\mathscr{L}(e)}{\longrightarrow} \mathscr{L}(G) \stackrel{\mathscr{L}(f)}{\longrightarrow} \mathscr{L}(H)
$$

is an exact sequence of topological Lie algebras. 
(ii) The subgroup $\langle\exp \mathscr{L}(G)\rangle$ generated by the image of the exponential function is dense in the identity component $G_{0}$ of $G$.

(See [9, Theorem 4.20 and Corollary 4.22(i)].)

Theorem 2.3 has various fairly immediate consequences.

COROLLARY 2.4. (i) If $N$ is a closed normal subgroup of a pro-Lie group $G$, then the quotient morphism $q: G \rightarrow G / N$ induces a map $\mathscr{L}(q): \mathscr{L}(G) \rightarrow \mathscr{L}(G / N)$ which is a quotient morphism with kernel $\mathscr{L}(N)$. Accordingly there is a natural isomorphism $X+\mathscr{L}(N) \mapsto \mathscr{L}(f)(X): \mathscr{L}(G) / \mathscr{L}(N) \rightarrow \mathscr{L}(G / N)$.

(ii) Let $G$ be a pro-Lie group. Then $\{\mathscr{L}(N) \mid N \in \mathscr{N}(G)\}$ converges to zero and is cofinal in the filter $\mathscr{I}(\mathscr{L}(G))$ of all ideals $\mathrm{i}$ such that $\mathscr{L}(G) / \mathrm{i}$ is finite dimensional. Furthermore, $\mathscr{L}(G)$ is the projective limit $\lim _{N \in \mathcal{N}(G)} \mathscr{L}(G) / \mathscr{L}(N)$ of a projective system of bonding morphisms and limit maps, all of which are quotient morphisms, and there is a commutative diagram

$$
\begin{aligned}
& \mathscr{L}(G) \stackrel{\mathscr{L}\left(\gamma_{G}\right)}{\longrightarrow} \mathscr{L}\left(G_{\mathcal{N}(G)}\right)=\mathscr{L}\left(\lim _{N \in \mathcal{N}(G)} \frac{G}{N}\right) \cong \lim _{N \in \mathcal{N}(G)} \frac{\mathscr{L}(G)}{\mathscr{L}(N)} \\
& \exp _{G} \downarrow \quad \exp _{G_{\mathcal{N}(G)} \downarrow} \downarrow \mid \lim _{N \in \mathcal{N}(G) \exp _{G / N}} \\
& G \quad \underset{\gamma_{G}}{\longrightarrow} G_{\mathcal{N}(G)}=\lim _{N \in \mathcal{N}(G)} G / N .
\end{aligned}
$$

(See $[9$, Corollary 4.21$]$.

For the proof of our Open Mapping Theorem it will be important to know that $\mathscr{L}(f)$ can be identified to be a quotient morphism even in cases where there is no prior knowledge that $f$ is an open morphism, as in the following corollary in which, for a subset $S$ of a group we write $\langle S\rangle$ for the subgroup generated by $S$, and where for a topological group $G$ the symbol $G_{0}$ denotes the connected component of the identity of $G$.

COROLLARY 2.5. (i) For a pro-Lie group $G$, the subgroup $\left(\exp _{G} \mathscr{L}(G)\right)$ is dense in $G_{0}$, that is, $G_{0}=\overline{\left\langle\exp _{G} \mathscr{L}(G)\right\rangle}$.

(ii) A morphism $f: G \rightarrow H$ of pro-Lie groups induces a surjective (hence quotient) morphism $\mathscr{L}(f): \mathscr{L}(G) \rightarrow \mathscr{L}(H)$ if $f$ is surjective and $G$ is almost connected.

(See $[9$, Corollary $4.22(\mathrm{i}, \mathrm{ii})]$.

While passing to a quotient group from a pro-Lie group is fraught with difficulties, there are sensible sufficient conditions for a quotient group of a pro-Lie group to be a pro-Lie group.

THEOREM 2.6. Assume that $G$ is a pro-Lie group and $K$ is a closed normal subgroup. Then $G / K$ is a pro-Lie group if $G$ and $K$ are both almost connected. (See $[9$, Theorem 4.28(i)].) 
In the reverse direction, if $G$ is a topological group and $N$ is a complete normal subgroup such that $G / N$ is complete, then $G$ is complete (see for instance [14, page 225, Theorem 12.3] ). Thus from this general remark we have the following proposition.

PROPOSITION 2.7. A proto-Lie group $G$ is a pro-Lie group if it contains a complete normal subgroup $N$ such that $G / N$ is a pro-Lie group.

In particular, if $G$ fails to be a pro-Lie group then all normal subgroups $N \in \mathscr{N}(G)$ are incomplete.

In the remainder of this preparatory section we provide some pieces of information that are not directly accessible in [9] and therefore we include proofs.

LEMMA 2.8. Let $G$ be a group with a completion $\bar{G}$ and let $N$ be a closed normal subgroup of $G$. We assume $G \subseteq \bar{G}$ and let $\bar{N}$ be the closure of $N$ in $\bar{G}$. Then $G \cap \bar{N}=N$ and the completion map of $G / N$ factors through the inclusion map $g N \mapsto g \bar{N}: G / N \rightarrow \bar{G} / \bar{N}$.

Proof. We let $p: G \rightarrow G / N$ and $P: \bar{G} \rightarrow \bar{G} / \bar{N}$ be the quotient maps and $i: G \rightarrow \bar{G}$ the embedding. Define $\eta: G / N \rightarrow \bar{G} / \bar{N}$ by $\eta(g N)=g \bar{N}$. To verify that $\eta$ factors as asserted, let $H$ be the completion of $G / N$ and $f: G / N \rightarrow H$ the completion map. Then $f \circ p: G \rightarrow H$ extends to a unique morphism $\gamma: \bar{G} \rightarrow H$ and, since $N=\operatorname{ker} p \subseteq f \circ p \subseteq \operatorname{ker} \gamma$, we have $\bar{N} \subseteq \operatorname{ker} \gamma$. So $\gamma$ factors uniquely in the form $\gamma=F \circ P$ with a unique morphism $F: \bar{G} / \bar{N} \rightarrow H$. Then $F \circ \eta \circ p=F \circ P \circ i=\gamma \circ i=f \circ p$ and since $p$ is an epimorphism, $f=F \circ \eta$. This completes the proof that $\eta$ factors as claimed. In particular, $\eta$ is injective and so $1=\operatorname{ker} \eta=(G \cap \bar{N}) / N$ and thus $G \cap \bar{N}=N$.

The following is a portion of Theorem 1.29 of [9] and belongs to the general theory of projective limits of topological groups.

LEMMA 2.9. Let $\mathscr{N}$ be a filter basis of closed normal subgroups of a topological group $G$ and set $G_{\mathcal{N}}=\lim _{N \in \mathcal{N}} G / N$. Let $v_{N}: G_{\mathscr{N}} \rightarrow G / N$ denote the limit map and $q_{N}: G \rightarrow G / N$ the quotient morphism. Write $\widetilde{N}=\operatorname{ker} v_{N}$.

(i) There is a morphism $\gamma=\gamma_{G, \mathcal{N}}: G \rightarrow G_{\mathcal{N}}$, given by $\gamma(g)=(g N)_{N \in \mathscr{N}}$, whose kernel is $\bigcap \mathscr{N}$ and whose image is dense.

(ii) $v_{N} \circ \gamma=q_{N}: G \rightarrow G / N$ for all $N \in \mathscr{N}$.

(iii) $v_{N}: G_{\mathcal{N}} \rightarrow G / N$ is a quotient map, that is, it induces an isomorphism $G_{\mathscr{N}} / \tilde{N} \rightarrow G / N$. Moreover, $\tilde{N}=\overline{\gamma(N)}$.

(iv) $G_{\mathcal{N}} \cong \lim _{N \in \mathscr{N}} G_{\mathscr{N}} / \tilde{N}$ and the filter basis $\{\tilde{N}: N \in \mathscr{N}\}$ converges to 1 in $G_{\mathcal{N}}$. 
PROOF. (i) From the definition of the limit it follows that $(g N)_{N \in \mathscr{N}} \in G_{\mathscr{N}}$. The function $\gamma$ is therefore well-defined; it is a morphism of groups and since $q_{N}=(g \mapsto g N): G \rightarrow G / N$ is continuous for each $N$ it follows that $\gamma$ is continuous. It is clear that $g \in \operatorname{ker} \gamma$ if and only if $g \in N$ for all $N \in \mathscr{N}$. Thus $\operatorname{ker} \gamma=\bigcap \mathscr{N}$. Now we must show that $\operatorname{im} \gamma$ is dense in $G_{\mathscr{N}}$. For this purpose let $\widetilde{g}=\left(g_{N} N\right)_{N \in \mathscr{N}} \in G_{\mathscr{N}}$ and let $U_{\mathscr{N}}$ be an identity neighborhood in $G_{\mathscr{N}}$. By [9, Theorem 1.27(i)], we find an identity neighborhood $U$ in $G$ and an $M \in \mathscr{N}$ such that $\nu_{M}^{-1}(U M / M) \subseteq U_{\mathcal{N}}$. Now $\gamma\left(g_{M}\right)=\left(g_{M} N\right)_{N \in \mathcal{N}}$ and

$$
v_{M}\left(\gamma\left(g_{M}\right)^{-1} \widetilde{g}\right)=v_{M}\left(\left(g_{M}^{-1} g_{N} N\right)_{N \in \mathscr{N}}\right)=g_{M}^{-1} g_{M} M=M,
$$

the identity of $G / M$. Thus $\gamma\left(g_{M}\right) \in \tilde{g} \tilde{M} \subseteq \tilde{g} U_{\mathscr{N}}$.

(ii) This is straightforward from the definitions.

(iii) From (ii) it follows that $v_{N}$ is surjective; hence $v_{N}$ induces a bijective morphism $v_{N}^{\prime}: G_{\mathcal{N}} / \tilde{N} \rightarrow G / N$ given by $v_{N}^{\prime}\left(\left(g_{M} M\right)_{M \in \mathcal{N}} \widetilde{N}\right)=g_{N} N$. Now the morphism $\widetilde{\nu}_{N} \circ \gamma: G \rightarrow G_{\mathscr{N}} / \widetilde{N}$, where $\widetilde{\nu}_{N}: G_{\mathscr{N}} \rightarrow G_{\mathscr{N}} / \tilde{N}$ is the quotient morphism, has kernel $\left\{g \in G \mid(g M)_{M \in \mathcal{N}} \in \tilde{N}\right\}=\{g \in G \mid g N=N\}=N$ and thus induces a morphism of topological groups $\gamma_{N}: G / N \rightarrow G_{\mathcal{N}} / \tilde{N}$ mapping $g N$ to $(g M)_{M \in \mathscr{N}} \tilde{N}$. One verifies at once that $v_{N}^{\prime}$ and $\gamma_{N}$ are inverses of each other. Hence $v_{N}^{\prime}$ is an isomorphism of topological groups and consequently $v_{N}$ is a quotient morphism. Since ker $\nu_{N}$ is closed and contains $\gamma(N)$ we have $\overline{\gamma(N)} \subseteq \operatorname{ker} \nu_{N}$.

An element $x=\left(g_{M} M\right)_{M \in \mathcal{N}} \in G_{\mathscr{N}}$ is in $\overline{\gamma(N)}$ if and only if there is a net $\left(g_{j}\right)_{j \in J}$ that is finally in $N$ and such that $x=\lim _{j \in J}\left(g_{j} M\right)_{M \in \mathscr{N}}$. If a $\left(g_{M} M\right)_{M \in \mathscr{N}} \in \widetilde{N} \subseteq G_{\mathscr{N}}$, $g_{N} \in N$ is given then for a fixed $P \in \mathscr{N}$ the net $\left(g_{M} P\right)_{M \in \mathcal{N}}$ in $G / P$ is finally constant, since $g_{R} \in g_{P} P$ for $R \subseteq P \in \mathscr{N}$. Also for $M \subseteq N$ we have $g_{M} \in g_{N} N \in N$. Thus $J=\mathscr{N},\left(g_{M}\right)_{M \in \mathscr{N}}$ yields such a net $\left(g_{j}\right)_{j \in J}$.

(iv) This follows from [9, Theorem 1.27].

We apply these facts directly to the situation of a proto-Lie group $G$.

PROPOSITION 2.10. Let $G$ be a proto-Lie group and $\mathscr{N}(G)$ the filter of closed normal co-Lie subgroups, converging to 1 . The limit $G_{\mathscr{N}(G)}$ is the completion $\bar{G}$ of $G$, and we consider $G$ as a dense subgroup of $\bar{G}$. For $N \in \mathscr{N}(G)$, let $\bar{N}$ be the closure of $N$.

(i) $\bar{N}$ is the kernel of the limit morphism $\bar{G} \rightarrow G / N$ and induces an isomorphism $\epsilon_{N}: \bar{G} / \bar{N} \rightarrow G / N$ which is inverted by $g N \mapsto g \bar{N}$. Accordingly, $\bar{G} \cong \lim _{N \in \mathcal{N}(G)} \bar{G} / \bar{N}$.

(ii) For each $N \in \mathscr{N}(G)$ one has $\bar{G}=G \bar{N}$ and $N=G \cap \bar{N}$. The Second Isomorphism Theorem holds and $\phi: G /(G \cap \bar{N}) \rightarrow G \bar{N} / \bar{N}$ where $\phi(g(G \cap \bar{N}))=g \bar{N}$ is an isomorphism of topological groups. 
(iii) The quotient morphism $\bar{G} / \bar{N}_{0} \rightarrow \bar{G} / \bar{N}$ induces an isomorphism

$$
\lim _{N \in \mathscr{N}(G)} \bar{G} / \bar{N}_{0} \rightarrow \lim _{N \in \mathscr{N}(G)} \bar{G} / \bar{N} \cong \bar{G}
$$

(iv) For $N \in \mathscr{N}(G)$ we have $N_{0}=G \cap \overline{N_{0}}=N \cap \overline{N_{0}}$, and the completion morphism of $G / N_{0}$ factors through the natural morphism $G / N_{0} \rightarrow \bar{G} / \overline{N_{0}}$. So

$$
\lim _{N \in \mathscr{N}(G)} G / N_{0} \rightarrow \lim _{N \in \mathcal{N}(G)} \bar{G} / \overline{N_{0}}
$$

is a dense embedding.

(v) If $\mathscr{L}(G)=\mathscr{L}(\bar{G})$, then $\overline{N_{0}}=\bar{N}_{0}$ and $\bar{G} / \overline{N_{0}}=\bar{G} / \bar{N}_{0}$.

For each $N \in \mathscr{N}(G)$, set $\mathfrak{n}=\mathscr{L}(N) ;$ then $\operatorname{dim} \mathfrak{g} / \mathfrak{n}<\infty$ and $\{\mathfrak{n}: N \in \mathscr{N}(G)\}$ is cofinal in id $\mathfrak{g}$, the filter basis of cofinite dimensional ideals of the pro-Lie algebra $\mathfrak{g}=\mathscr{L}(\bar{G})$.

(vi) In the circumstances of (v), if $G$ is almost connected then $\bar{G} / \bar{N}_{0}$ is a locally compact almost connected group.

PROOF. (i) is a direct consequence of Lemma 2.9.

(ii) By (i), the morphism $g N \mapsto g \bar{N}: G / N \rightarrow \bar{G} / \bar{N}$ is an isomorphism. Thus, firstly, it is surjective, whence $\bar{G}=G \bar{N}$, and secondly, it is injective, whence $G \cap \bar{N}=N$; thirdly, it is an isomorphism of topological groups, and agrees with $\phi$. Therefore $\phi$ is an isomorphism.

(iii) is a consequence of Lemma 2.9 .

(iv) By Lemma 2.8, $N_{0}=G \cap \overline{N_{0}}$ and the completion morphism of $G / N_{0}$ factors through $G / N_{0} \rightarrow \bar{G} / \overline{N_{0}}$. Hence the morphism

$$
\left(g_{N} N_{0}\right)_{N \in \mathcal{N}(G)} \mapsto\left(g_{N} \overline{N_{0}}\right)_{N \in \mathcal{N}(G)}: \lim _{N \in \mathcal{N}(G)} G / N_{0} \rightarrow \lim _{N \in \mathcal{N}(G)} \bar{G} / \overline{N_{0}}
$$

is a dense embedding.

(v) Assume that $G$ and $\bar{G}$ have the same exponential function. Let $N \in \mathscr{N}(G)$ and let $q_{N}: G \rightarrow G / N$ denote the quotient map. We set $\mathfrak{n}=\mathscr{L}(N)$. Since $G / N$ is a Lie group and $\mathscr{L}$ preserves kernels,

$$
\mathrm{n}=\mathscr{L}\left(\operatorname{ker} q_{N}\right)=\operatorname{ker} \mathscr{L}\left(q_{N}\right) \text { for } \quad \mathscr{L}\left(q_{M}\right): \mathscr{L}(G) \rightarrow \mathscr{L}(G / N),
$$

the quotient $g / \mathfrak{n}$ is finite dimensional. At this point we use the crucial hypothesis $\mathfrak{g}=\mathscr{L}(G)=\mathscr{L}(\bar{G})$ and note that $\exp _{G} \mathfrak{n}=\exp _{\bar{G}} \mathfrak{n} \subseteq \bar{G}$. Now $\bar{N}$ is a closed subgroup of the pro-Lie group $\bar{G}$ and is therefore a pro-Lie group by the Closed Subgroup Theorem [9, Theorem 3.35]. Hence $\overline{\left\langle\exp _{\bar{G}} \mathfrak{n}\right\rangle}=\bar{N}_{0} \subseteq \bar{N} \subseteq \bar{G}$. Also we observe that $\left\langle\exp _{G} n\right\rangle \subseteq N_{0} \subseteq \bar{N}_{0}$. From these pieces of information we derive

$$
\overline{N_{0}}=\bar{N}_{0}, \quad \text { and, accordingly, } \quad \bar{G} / \overline{N_{0}}=\bar{G} / \bar{N}_{0} .
$$


Also,

$$
\bar{G}=\lim _{N \in \mathcal{N}(G)} G / N \cong \lim _{N \in \mathcal{N}(G)} \bar{G} / \bar{N}
$$

shows that $\{\bar{N}: N \in \mathscr{N}(G)\}$ is cofinal in $\mathscr{N}(\bar{G})$ and thus $\{\mathrm{n}: N \in \mathscr{N}(G)\}$, $\mathfrak{n}=\mathscr{L}(N)=\mathscr{L}(G \cap \bar{N})=\mathscr{L}(G) \cap \mathscr{L}(\bar{N})=\mathscr{L}(\bar{N})$, is cofinal in id $\mathfrak{g}$.

(vi) Recall from [9, Theorem 9.44] that an almost connected pro-Lie group with finite dimensional Lie algebra is locally compact metric. Thus if $G$ is almost connected, then the morphism $g \mapsto g \bar{N}_{0}: G \rightarrow \bar{G} / \bar{N}_{0}$ has a dense image. Hence $\bar{G} / \bar{N}_{0}$ is almost connected by Lemma 3.2 below and thus is locally compact.

It is very useful to have certain purely algebraic information on pro-Lie groups. Recall that a divisible group is one in which the equation $x^{n}=g$ has a solution for every group element $g$ and every natural number $n$.

PROPOSITION 2.11. Let $G$ be a connected pro-Lie group. Then

(i) every $g \in G$ that is contained in a compact subgroup (which is the case if $g$ has finite order) is contained in a divisible subgroup;

(ii) the group $G$ is algebraically generated by the union of all divisible subgroups;

(iii) $G$ has no normal subgroups of finite index.

PROOF. (i) By [9, Theorem 12.77], every compact subgroup of $L$ is contained in a connected compact subgroup. Compact connected groups are divisible (see, for example, [5, Theorem 9.35]).

(ii) By [9, Theorem 12.65], $G$ is the product of a compact subgroup and the subgroup $\left\langle\exp _{G} \mathfrak{g}\right\rangle$ that is algebraically generated by all one-parameter subgroups. This, together with (i), shows that $G$ is algebraically generated by divisible subgroups.

(iii) In every group $G$, the subgroup $D(G)$ algebraically generated by all divisible subgroups is a characteristic subgroup such that for any homomorphisms $f: G \rightarrow H$ we have $f(D(G)) \subseteq D(H)$. Thus if $G$ is a connected pro-Lie group then by (ii) we have $G=D(G)$ and so for any homomorphism $f: G \rightarrow H$ the image $f(G)$ is contained in $D(H)$. If $N$ is any normal subgroup of $G$, closed or not, then $G / N=D(G / N)$. If $G / N$ is finite then $D(G / N)=\{N\}$ and so $N=G$.

PROPOSITION 2.12. Let $C$ be a closed central totally disconnected subgroup of a connected pro-Lie group $L$. Then

(i) $\mathscr{N}(C)$ has a basis of subgroups $B$ so that $C / B$ is a direct product of a finite abelian group and a finitely generated free abelian group;

(ii) in particular, if $C \neq\{1\}$ then there is a prime number $p$ and a closed subgroup $B$ of $C$ such that $C / B \cong \mathbb{Z}(p)$;

(iii) if $L$ contains a subgroup $G$ such that $L=C G$ and $C \cap G=\{1\}$ then $C=\{1\}$. 
Proof. (i) Let $N \in \mathscr{N}(L)$. Then $\overline{C N} / N$ is a closed central subgroup of the Lie group $L / N$. By [9, Theorem 1.34(i)], $C$ is canonically isomorphic to $\lim _{N \in \mathscr{N}(L)} \overline{C N} / N$ where $\overline{C N} / N$ is discrete since $C$ is prodiscrete. Also, if $L$ is connected then $\overline{C N} / N$ is a closed central subgroup of the connected Lie group $L / N$. Hence it is a finitely generated abelian group and so $C N / N$ is also finitely generated abelian, that is, it is isomorphic to a direct product of finitely many cyclic groups. By [9, Theorem 1.34(iv)], we know that $C /(C \cap N) \cong C N / N$ as topological groups, and so there is a closed subgroup $B_{N}=C \cap N$ of $C$ such that $C / B_{N}$ is finitely generated discrete, and $\lim _{N \in \mathscr{N}(L)} B_{N}=\lim _{N \in \mathscr{N}(L)} N=1$.

(ii) Any direct product of cyclic groups has a quotient of prime order. Thus (ii) is a consequence of (i).

(iii) We suppose that $C \neq\{1\}$ and derive a contradiction. From (ii) we get an openclosed subgroup $B$ of $C$ such that $C / B=\mathbb{Z}(p)$. Since $L$ is algebraically the direct product $C \cdot G$ and $B$ is contained in $C$, the factor group $L / B$ is algebraically the direct product $(C / B) \cdot(G B / B)$. Hence, algebaically, $L / G B \cong(L / B) /(G B / B) \cong C / B$. Thus the connected pro-Lie group $L$ has a normal subgroup $G B$ of index $p$. This is impossible by Proposition 2.11(iii).

\section{Completing proto-Lie groups}

If $\bar{G} \supseteq G$ is the completion of a proto-Lie group $G$, one might surmise that we must have $\mathscr{L}(G)=\mathscr{L}(\bar{G})$. However this equality fails rather grotesquely, as we shall show presently by exhibiting appropriate examples. Therefore we introduce a convenient terminology.

DEFINITION 3.1. A proto-Lie group $P$ has a stable Lie algebra if $\mathscr{L}(\bar{P})=\mathscr{L}(P)$ for its completion $\bar{P}$.

Another seemingly reasonable conjecture would be that every bijective morphism $f: G \rightarrow H$ from a connected proto-Lie group $G$ to a pro-Lie group $H$ must be an isomorphism. Unfortunately, this conjecture is also false.

These things we leam, among other things, from the following examples, which we find useful to keep in mind. Since their discussion involves real vector spaces and their vector space dimensions, we preface it with the following remarks which are less obvious than their proofs.

REMARK 1. (a) Let $W$ be a real vector space of infinite dimension $\aleph$, that is, $\operatorname{dim}_{\mathbb{R}} W=\aleph$. Then $\operatorname{dim}_{\mathbb{R}} W^{*}=2^{\kappa}$ and $\operatorname{dim}_{\mathbb{R}} W^{* *}=2^{2^{*}}$.

(b) The additive group of any vector subspace of a weakly complete vector space is a proto-Lie group. 
PROOF. (a) Pick a basis $B$ of $W$; then card $B=\aleph$. Now the algebraic dual $W^{*}$ is given by $W^{*}=\operatorname{Hom}(W, \mathbb{R}) \cong \operatorname{Hom}\left(\mathbb{R}^{(B)}, \mathbb{R}\right) \cong \mathbb{R}^{B}$. Thus $\operatorname{dim}_{\mathbb{R}} W^{*}=2^{\aleph}$. (Indeed if $E=\mathbb{R} \otimes E_{\mathbf{Q}}$ for a $\mathbb{Q}$-vector space $E_{\mathbf{Q}}$ then $\operatorname{dim}_{\mathbb{R}} E=\operatorname{dim}_{\mathbf{Q}} E_{\mathbf{Q}}$ and if $\mathbb{Q}^{(X)}$ is any infinite dimensional vector space then $\operatorname{dim}_{\mathbf{Q}} \mathbb{Q}^{(X)}=\operatorname{card}(X)=\operatorname{card} \mathbb{Q}^{(X)}$. Now $\mathbb{R} \otimes \mathbb{Q}^{B}$ is isomorphic to a vector subspace of $\mathbb{R}^{B}$ and so

$$
\begin{aligned}
2^{\operatorname{card} B} & =\operatorname{card} \mathbb{Q}^{B}=\operatorname{dim}_{\mathbf{Q}} \mathbb{Q}^{B}=\operatorname{dim}_{\mathbb{R}} \mathbb{R} \otimes \mathbb{Q}^{B} \leq \operatorname{dim}_{\mathbb{R}} \mathbb{R}^{B} \leq \operatorname{card} \mathbb{R}^{B}=\left(2^{\aleph_{0}}\right)^{\operatorname{card} B} \\
& =2^{\aleph_{0} \operatorname{card} B}=2^{\operatorname{card} B},
\end{aligned}
$$

since $B$ is infinite.) Therefore $\operatorname{dim} W^{* *}=2^{2^{*}}$. Furthermore, if $W$ is a real vector space of infinite dimension $2^{\aleph}$ then $W \cong \mathbb{R}^{B}$ for a set $B$ of cardinality $\aleph$ because $\operatorname{dim}_{\mathbb{R}} \mathbb{B}^{\aleph}=\operatorname{dim}_{\mathbf{Q}} \mathbb{Q}^{\aleph}=2^{\aleph}$.

(b) Let $V$ be a weakly complete vector space and $E$ any vector subspace. Let $U_{E}$ be a neighborhood of 0 in $E$ and $U_{V}$ a neighborhood of 0 in $V$ such that $U_{E}=E \cap U_{V}$. Then there is a closed vector subspace $W$ contained in $U_{V}$ such that $\operatorname{dim} V / W<\infty$. Now $E \cap W \subseteq E \cap U_{V}=U_{E}$ and $E /(E \cap W) \cong(E+W) / W \subseteq V / W$. Since $(E+W) / W$ is a vector subspace of a finite dimensional vector space, $E /(E \cap W)$ is a finite dimensional vector space. It follows that $E$ is a proto-Lie group.

The following examples are now straightforward from the preceding remarks.

EXAMPLE 1. There is a connected proto-Lie group $G$ whose Lie algebra $\mathscr{L}(G)$ is algebraically isomorphic to $\mathbb{R}^{\aleph_{0}}$, while $\mathscr{L}(\bar{G}) \cong \mathbb{R}^{2^{2^{\aleph_{0}}}}$.

Proof. let $V$ be the vector space $\mathbb{R}^{N}$ and let $\theta_{V}: V \rightarrow V^{* *}$ be the natural morphism into its algebraic bidual, given by $\left\langle\theta_{v}(v), \omega\right\rangle=\langle\omega, v\rangle$ for $v \in V, \omega \in V^{*}$. Let $\bar{G}$ be the additive group of $V^{* *}$ with the weak-* topology. Then $\bar{G}$ is a pro-Lie group and $\mathscr{L}(\bar{G})=V^{* *}$. Thus $\mathscr{L}(\bar{G})=\mathbb{R}^{2^{2_{0}}}$. Now let $G=\theta_{V}\left(\mathbb{R}^{\mathbb{N}}\right)$ with the subgroup topology of $\bar{G}$. Then $G$ is a proto-Lie group with $\mathscr{L}(G)$ algebraically isomorphic to $\mathbb{R}^{\kappa_{0}}$.

EXAMPLE 2. Let $\omega: H \rightarrow G_{1}$ be a group homomorphism between connected pro-Lie groups such that the graph

$$
G \stackrel{\text { def }}{=}\{(h, \omega(h)): h \in H\} \subseteq \bar{G} \stackrel{\text { def }}{=} H \times G_{1}
$$

is a proper dense subgroup. Then $\left.f \stackrel{\text { def }}{=} \mathrm{pr}_{H}\right|_{G}: G \rightarrow H$ is a bijective but nonopen morphism which extends to a surjective open morphism $F \stackrel{\text { def }}{=} \operatorname{pr}_{H}: \bar{G} \rightarrow H$ of pro-Lie groups.

Specifically, let $\mathbb{K}$ denote either (a) $\mathbb{R}$, or (b) the discrete field $\mathbb{Z}(p)=\mathbb{Z} / p \mathbb{Z}$ for some prime $p$, and consider $H=\mathbb{K}^{N}$ with its product topology, which in case (a) is a 
pro-Lie group agreeing with $\mathscr{L}(H)$ (up to a natural isomorphism) and in case (b) is a compact totally disconnected vector space over the field of $p$ elements. Let $G_{1}=\mathbb{K}$ and let $\omega: H \rightarrow G_{1}$ be a discontinuous $\mathbb{K}$-linear form. Such a linear map certainly exists, since the topological dual $\widehat{\mathbb{K}^{N}}$ is isomorphic to $\mathbb{K}^{(\mathbb{N})} \cong \mathbb{R}^{\left(\mathbb{N}_{0}\right)}$, while the algebraic dual $\left(\mathbb{K}^{N}\right)^{*}$ is isomorphic to $\mathbb{K}^{2^{2^{\kappa_{0}}}}$. Now the graph $G$ of $\omega$ in $H \times G_{1}$ is a $\mathbb{K}$-hyperplane, being the image of $\mathbb{K}^{N} \times\{0\}$ under the linear automorphism $(x, y) \mapsto(x, y+\omega(x))$ of $\mathbb{K}^{\mathbb{N}} \times \mathbb{K}$. Since $\omega$ is discontinuous, $G$ is dense, since hyperplanes in a topological vector space are either closed or dense.

Case (a): $G=\mathscr{L}(G)$, as the additive group of a vector subspace of a weakly complete vector space, is a connected proto-Lie group which is not a pro-Lie group. $G$ does not have a stable Lie algebra. $H=\mathscr{L}(H)$ is a connected pro-Lie group. The morphism $f: G \rightarrow H$ is bijective and not open.

Case (b): $G$ is a precompact protofinite (hence proto-Lie) group but is not almost connected, while $H$ is an abelian compact totally disconnected, hence almost connected, group. By default, both $\mathscr{L}(G)$ and $\mathscr{L}(H)$ are singleton and so $G$ has a stable Lie algebra and $\mathscr{L}(f)$ is an isomorphism. The morphism $f: G \rightarrow H$ is continuous, bijective and not open.

It is worth noting in passing that the construction in Example 2 yields an interesting example in the world of finite dimensional Lie groups: we may take $H=G_{1}=\mathbb{R}$ and find a bijective $\mathbb{Q}$-linear map $\omega: \mathbb{R} \rightarrow \mathbb{R}$ that is not a multiplication by a real number. This can even be done in such a fashion that the graph $G$ of $\omega$ is a connected subgroup of $\mathbb{R}^{2}$. (For the existence of such an $f$ see [11].) Here $G$ is a connected abelian topological group which is arcwise totally disconnected, causing $\mathscr{L}(G)$ to be zero; its completion is $\mathbb{R}^{2}$, but it is certainly not a proto-Lie group because it has no small subgroups and is not a Lie group. We have $\mathscr{L}(\bar{G}) \cong \mathbb{R}^{2}, \mathscr{L}(H) \cong \mathbb{R}$.

Nevertheless, if $G$ is a proto-Lie group with a stable Lie algebra, in contrast with Example 2(a), then for each subgroup $H$ of $\bar{G}$ one has

$$
\mathscr{L}(H)=\mathscr{L}(G \cap H) \quad \text { and } \quad\langle\exp \mathscr{L}(H)\rangle \subseteq G
$$

In particular, the subgroup $\left\langle\exp _{\bar{G}} \mathscr{L}(G)\right\rangle$ of $\bar{G}$ having the full Lie algebra

$$
\mathscr{L}\left(\left\langle\exp _{\bar{G}}\right\rangle\right)=\mathscr{L}(G)=\mathscr{L}(\bar{G})
$$

is contained in $G$. (For information on analytic subgroups of pro-Lie groups, see [ 9 , Chapter 9].)

LEMMA 3.2. Let $f: G \rightarrow H$ be a morphism of topological groups and assume, firstly, that $G$ is almost connected and, secondly, that $f(G)$ is dense in $H$. Then $H$ is almost connected. 
PROOF. Since $f\left(G_{0}\right)$ is connected and contains the identity, $f\left(G_{0}\right) \subseteq H_{0}$ and therefore the morphism $\phi: G / G_{0} \rightarrow H / H_{0}$ given by $\phi\left(g G_{0}\right)=f(g) H_{0}$ is welldefined. By assumption, $G / G_{0}$ is compact. So the continuous image $\phi\left(G / G_{0}\right)$ is compact and thus, since $H / H_{0}$ is Hausdorff, is closed in $H / H_{0}$. Since $f(G)$ is dense in $H$, it follows that $\phi\left(G / G_{0}\right)$ is dense in $H / H_{0}$. Therefore $H / H_{0}=\phi\left(G / G_{0}\right)$ and so $H / H_{0}$ is compact.

Thus if $G$ is an almost connected proto-Lie group then $\bar{G}$ is an almost connected proLie group. If $f: G \rightarrow H$ is a morphism of a topological group $G$ with a completion $\bar{G} \supseteq G$ into a complete topological group $H$ then there is a unique extension of $f$ to a morphism $F: \bar{G} \rightarrow H$, whose kernel we shall consistently denote by $C$. The following Lemma is one crucial ingredient in the proof of the Open Mapping Theorem.

LEMMA 3.3. Let $G$ be a proto-Lie group with a stable Lie algebra and assume that $f: G \rightarrow H$ is a bijective morphism onto a complete group and that $\bar{G}$ is connected. Then $G$ is a pro-Lie group.

Proof. Let $F: \bar{G} \rightarrow G$ the unique extension of $f$ with kernel $C \stackrel{\text { def }}{=}$ ker $f$. Since $G$ has a stable Lie algebra, $\mathscr{L}(C)=\mathscr{L}(\operatorname{ker} F)=\operatorname{ker} \mathscr{L}(F)=\operatorname{ker} \mathscr{L}(f)=\{0\}$, and thus $C$ is totally disconnected normal in the connected group $\bar{G}$ and is, therefore, central. If $\gamma: G \rightarrow \bar{G}$ is the embedding morphism, then $\phi: \bar{G} \rightarrow \bar{G}, \phi=\gamma \circ f^{-1} \circ F$ is an idempotent morphism of groups since $f^{-1} \circ F \circ \gamma=\mathrm{id}_{G}$. Note that im $\phi=G$ and $\operatorname{ker} \phi=\operatorname{ker} F=C$. Thus $\bar{G}=C G$ with $C \cap G=\{1\}$ is a bijective continuous homomorphic image of the direct product $C \times G$ under the morphism $(c, g) \mapsto c g$. Now Proposition 1.12(iii) applies and shows that $C=\{1\}$. Thus $F$ is injective, that is, $\phi$ is bijective and thus $G=\bar{G}$.

Note that $\bar{G}$ is certainly connected if $G$ is connected, which we usually assume. A proof of the openness of $f$ will be one of our targets.

\section{The Proof of the Open Mapping Theorem}

We now propose to prove the following main result.

THEOREM 4.1 (Open Mapping Theorem for Pro-Lie Groups). Let $f: G \rightarrow H$ be a surjective morphism from an almost connected pro-Lie group onto a pro-Lie group. Then $f$ is open. 
Towards a proof, we consider the canonical decomposition diagram for $f$ :

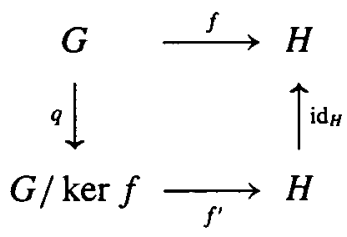

with $q$ being the quotient morphism and $f^{\prime}$ the induced bijective morphism defined by $f^{\prime}(g \operatorname{ker} f)=f(g)$.

By Lemma 3.2, the quotient $G / \operatorname{ker} f$ is almost connected since $G$ is almost connected. By Theorem 2.2, $G / \operatorname{ker} f$ is a proto-Lie group. By Theorem 2.3 the embedding of $G / \operatorname{ker} f$ into its completion $\overline{G / \operatorname{ker} f}$ induces an isomorphism $\mathscr{L}(G / \operatorname{ker} f) \rightarrow \mathscr{L}(\overline{G / \operatorname{ker} f})$, so $G / \operatorname{ker} f$ has a stable Lie algebra.

If we can show that the bijective morphism $f^{\prime}: G / \operatorname{ker} f \rightarrow H$ from an almost connected proto-Lie group with stable Lie algebra onto a pro-Lie group is an isomorphism, we thereby prove that $f$ is open.

Thus we need the following Lemma.

LEMMA 4.2. If $f: G \rightarrow H$ is a bijective morphism from an almost connected protoLie group with stable Lie algebra onto a pro-Lie group then $f$ is an isomorphism.

Example 2(b) comes dangerously close to being a counterexample to Lemma 4.2, but in that example $G$ is not almost connected, though both $\bar{G}$ and $H$ are since they are compact and totally disconnected.

Before we prove Lemma 4.2, let us reduce the task by showing that if Lemma 4.2 is true for a connected group $G$ (and consequently a connected $H$ ), then it is true as stated above for almost connected groups.

Thus let $f: G \rightarrow H$ be as in Lemma 4.2. Let $P=f^{-1}\left(H_{0}\right)$. Then $G_{0} \subseteq P$. Thus $P$ is an almost connected proto-Lie group and $\left.f\right|_{P}: P \rightarrow H_{0}$ is bijective since $f$ is bijective. We claim that $P$ is connected and therefore equals $G_{0}$. If not, then $P$ has an open normal proper subgroup $Q$ of finite index since $G / G_{0}$ is profinite as $G$ is almost connected. But then, $f$ being surjective, $f(Q)$ is a normal subgroup of finite index in $H_{0}$. By Proposition 2.11(iii), this implies $f(Q)=H_{0}$ and, since $f$ is injective, $Q=P$ which contradicts the assumption that $Q$ was a proper subgroup.

Now we assume that Lemma 4.2 is true if $G$ and $H$ are connected. Then $\left.f\right|_{G_{0}}: G_{0} \rightarrow H_{0}$ is an isomorphism of topological groups, so $G_{0} \cong H_{0}$ is a proLie group. Then, since $G / G_{0}$ is compact, by Proposition 2.7, $G$ is a pro-Lie group.

Therefore, $f: G \rightarrow H$ is a bijective morphism between almost connected groups, such that $\left.f\right|_{G_{0}}: G_{0} \rightarrow H_{0}$ is an isomorphism. If $U$ is an open neighborhood of the identity in $G$, we find a normal subgroup $N \in \mathscr{N}(G)$ such that $M \stackrel{\text { def }}{=} N \cap G_{0} \in \mathscr{N}\left(G_{0}\right)$. 
It is no loss of generality to assume that $U N=U$ (see, for example, [9, Theorem 1.27(i)].) The factor group $G / M$ is now a locally compact almost connected and hence $\sigma$-compact group. Since $\left.f\right|_{G_{0}}: G_{0} \rightarrow H_{0}$ is an isomorphism, $H_{0} / f(M)$ is isomorphic to $G_{0} / M$ and is therefore a Lie group, whence $H / f(M)$ is locally compact. Thus the induced map $f_{M}: G / M \rightarrow H / f(M)$ is open by Theorem $\mathrm{A}$, the classical Open Mapping Theorem for Locally Compact Groups. Hence $f_{M}(U / M)=f(U) / f(M)$ is open in $H / f(M)$ and so $f(U)$ is open in $H$. Thus $f$ is open and therefore an isomorphism.

After this reduction, we concentrate on the following assertion.

Let $f: G \rightarrow H$ be a bijective morphism from a connected proto-Lie group with stable Lie algebra onto a pro-Lie group, then $f$ is an isomorphism.

From Lemma 3.3 we know that $G$ is a pro-Lie group. Thus we have to prove the following.

LEMMA 4.3. If $f: G \rightarrow H$ is a bijective morphism between connected pro-Lie groups and assume that $G$ has a stable Lie algebra. Then $f$ is an isomorphism.

A proof of this lemma will prove the Open Mapping Theorem for Pro-Lie Groups (Theorem D of the Introduction).

Thus for a proof of Lemma 4.3 we need to establish that a bijective morphism between two connected pro-Lie groups is an isomorphism.

LEMMA 4.4. Let $G$ be a connected proto-Lie group and $f: G \rightarrow H$ a bijective morphism onto a pro-Lie group. Then the following statements are true.

(i) For all $N \in \mathscr{N}(H)$ the normal subgroup $M \stackrel{\text { def }}{=} f^{-1}(N)$ is in $\mathscr{N}(G)$ and $\phi_{N}: G / M \rightarrow H / N$ given by $\phi_{N}(g M)=f(g) N$ is an isomorphism of Lie groups.

$$
f^{-1}(\mathscr{N}(H))=\left\{f^{-1}(N): N \in \mathscr{N}(H)\right\}
$$

is a faithful copy of the filter basis $\mathscr{N}(H)$ inside $\mathscr{N}(G)$.

(ii) If we set $M^{*}=f^{-1}(\overline{f(M)})$ for $M \in \mathscr{N}(G)$ then

$$
N \stackrel{\text { def }}{=} f\left(M^{*}\right)=\overline{f(M)} \in \mathscr{N}(H),
$$

and $M \mapsto M^{*}: \mathscr{N}(G) \rightarrow \mathscr{N}(G)$ is a well defined order retraction of $\mathscr{N}(G)$ onto $f^{-1}(\mathscr{N}(H))$ such that $M \subseteq M^{*}=M^{* *}$ and that $\phi_{M}: G / M^{*} \rightarrow H / N$ given by $\phi_{M}\left(g M^{*}\right)=f(g) N$ is an isomorphism of Lie groups. Accordingly, there is an isomorphism

$$
\phi: \lim _{M \in \mathcal{N}(G)} G / M^{*} \rightarrow H,
$$

where $\left.\phi\left(\left(g_{M} M^{*}\right)_{M \in \mathcal{N}(G)}\right)\right)=\lim _{M \in \mathcal{N}(G)} f\left(g_{M}\right)$, as limit of a Cauchy sequence in $H$ and where $\phi^{-1}(h)=\left(f^{-1}(h) M\right)_{M \in \mathscr{N}(G)}$. 
PRoof. (i) Consider an $N \in \mathscr{N}(H)$. Let $V$ be an open neighborhood of the identity in $H$ containing $N$ and such than $V N=V$ and $V / N$ contains no subgroups other that the singleton one. Since $f$ is continuous, $U \stackrel{\text { def }}{=} f^{-1}(V)$ is a neighborhood of the identity in $G$. Set $M \stackrel{\text { def }}{=} f^{-1}(N)$. Then $U M=U$ and $U M / M$ contains no subgroup other than the singleton one. Since $\lim \mathscr{N}(G)=1$ in $G$ there is a $P \in \mathscr{N}(G)$ such that $P \subseteq U$. Then $P M / M$ is a subgroup of $G / M$ contained in $U / M$ and thus agrees with $M / M$, that is, $P M=M$, and so $P \subseteq M$. Therefore we have a surjective morphism of topological groups $\phi_{N P}: G / P \rightarrow H / N$ given by $\phi_{N P}(g P)=f(g) N$.

By the definition of $\mathscr{N}(G)$ we know that $G / P$ is a Lie group; it is almost connected by Lemma 3.2 since $G$ is almost connected. Thus $G / P$ is a locally compact group which is the union of a countable collection of compact subsets. Also, $H / N$ is a Lie group since $N \in \mathscr{N}(H)$. Hence it is locally compact. Thus the Open Mapping Theorem for Locally Compact Groups (see Theorem A) applies and shows that $\phi_{N P}$ is open. Since $M / P=\operatorname{ker} \phi_{N P}$, we know that $G / M \cong(G / P) /(M / P) \cong H / N$ is a Lie group. Therefore $M \in \mathscr{N}(G)$. Thus

$$
f^{-1}(\mathscr{N}(H)) \subseteq \mathscr{N}(G)
$$

and $\phi_{N}: G / M \rightarrow H / N$ given by $\phi_{N}(g M)=f(g) N$ is an isomorphism. This completes the proof of (i).

(ii) For $M \in \mathscr{N}(G)$ set $M^{*}=f^{-1}(\overline{f(M)})$. Then $f\left(M^{*}\right)=\overline{f(M)}$ and we define $N=\overline{f(M)}$. Then $M^{*}$ is a closed normal subgroup in $G$ by the continuity of $f$, and $M \subseteq M^{*}=M^{* *}$. So $G / M^{*} \cong(G / M) /\left(M^{*} / M\right)$ is a quotient of a Lie group and is, therefore, a Lie group. Thus we have $M^{*} \in \mathscr{N}(G)$ and so there is a bijective morphism $\psi_{M}: G / M^{*} \rightarrow H / N$ from a Lie group $G / M^{*}$ to a proto-Lie group $H / N$. Since $G$ is connected, so is $H$. Hence Theorem 2.6 applies to show that $H / N_{0}$ is a pro-Lie group. By Theorem 2.3, we have $\mathscr{L}(H / N)=\mathfrak{h} / \mathfrak{n}$, where $\mathfrak{n}=\mathscr{L}(N)$, and $\mathscr{L}(f)(\mathfrak{m}) \subseteq \mathscr{L}(f)\left(\mathfrak{m}^{*}\right)=\mathfrak{n}$.

Hence $\mathfrak{h} / \mathfrak{n}$ is a homomorphic image of $\mathfrak{g} / \mathfrak{m}$ and thus is finite dimensional. Thus $H / N_{0}$ is a finite dimensional pro-Lie group and so is locally compact by Proposition 2.7. As a quotient of a locally compact group, $H / N=\left(H / N_{0}\right) /\left(N / N_{0}\right)$ is locally compact as well. Then the bijective morphism from a locally compact almost connected group $G / M^{*}$ to a locally compact group $H / N$ is open by the Open Mapping Theorem for Locally Compact Groups (Theorem A), and therefore is an isomorphism. This means that $H / N \cong G / M^{*}$ is a Lie group and thus $N \in \mathscr{N}(H)$.

For $M \in \mathscr{N}(G)$, the natural isomorphism $\phi_{M}: G / M^{*} \rightarrow H / f\left(M^{*}\right)$, induces an isomorphism $\phi$ which is the composition of

$$
\lim _{M \in \mathcal{N}(G)} G / M^{*} \rightarrow \lim _{M \in \mathcal{N}(G)} H / f\left(M^{*}\right)=\lim _{N \in \mathcal{N}(H)} H / N
$$


For $\left(g_{M} M^{*}\right)_{M \in \mathscr{N}(G)} \in \lim _{M \in \mathscr{N}(G)} G / M^{*}$ the net $\left(f\left(g_{M}\right)\right)_{M \in \mathscr{N}(G)}$ in $H$ is a Cauchy net and thus has a limit $\phi\left(\left(g_{M} M^{*}\right)_{M \in \mathcal{N}(G)}\right)$, giving an isomorphism

$$
\phi: \lim _{M \in \mathcal{N}(G)} G / M^{*} \rightarrow H
$$

whose inverse is given by $\phi^{-1}(h)=\left(f^{-1}(h) M\right)_{M \in \mathcal{N}(G)}$. This proves (ii).

LEMMA 4.5. Let $G$ be a connected proto-Lie group with stable Lie algebra and $f: G \rightarrow H$ a bijective morphism onto a pro-Lie group. Then $\mathscr{L}(f): \mathfrak{g} \rightarrow \mathfrak{h}$ where $\mathfrak{g}=\mathscr{L}(G)$ and $\mathfrak{h}=\mathscr{L}(H)$, is an isomorphism of pro-Lie algebras.

PROOF. We are assuming now that $\mathscr{L}(G)=\mathscr{L}(\bar{G})$. Since $G$ is almost connected, $\bar{G}$ is almost connected by Lemma 3.2. Because $H$ is complete, the morphism $f: G \rightarrow H$ extends to a morphism $F: \bar{G} \rightarrow H$. As $f$ is bijective, $F$ is surjective. Then Corollary 2.5(ii) applies to show that $\mathscr{L}(F): \mathscr{L}(\bar{G}) \rightarrow \mathfrak{h}$ is a quotient morphism. If $\mathfrak{g}=\mathscr{L}(\bar{G})$, then this implies that $\mathscr{L}(f)=\left.\mathscr{L}(F)\right|_{\mathfrak{g}}$ is surjective and hence is a quotient morphism. Since $f$ is bijective, $\operatorname{ker} \mathscr{L}(f)=\{0\}$. It follows that $\mathscr{L}(f)$ is an isomorphism.

Now let $M \in \mathscr{N}(G)$ and $\mathfrak{m}=\mathscr{L}(M)$. Then $\{\mathfrak{m}: M \in \mathscr{N}(G)\}$ is cofinal in id $\mathfrak{g}$ by Proposition $2.10(\mathrm{vi})$. For $M \in \mathscr{N}(G)$ we let $\mathfrak{m}^{*}=\mathscr{L}\left(M^{*}\right)$. Then $\left\{\mathrm{m}^{*}: M \in \mathscr{N}(G)\right\}=\{\mathrm{n}: N \in \mathscr{N}(H)\}$ by Lemma 4.4. Since $\mathscr{L}(f)$ is an isomorphism by Lemma $4.5,\{\mathscr{L}(f)(\mathfrak{m})=\mathscr{L}(f(M)): M \in \mathscr{N}(G)\}$ is cofinal in id $\mathfrak{h}$, as is the set $\{\mathfrak{n}: N \in \mathscr{N}(H)\}$, where $\mathfrak{n}=\mathscr{L}(N)$. We conclude that $\left\{\mathfrak{m}^{*}: M \in \mathscr{N}(G)\right\}$ is cofinal in id $G$.

We now assume that $G$ is a connected pro-Lie group and recall from Theorem 2.3(ii) that $M_{0}=\left\langle\exp _{G} \mathfrak{m}\right\rangle$ and $\left(M^{*}\right)_{0}=\left\langle\exp _{G} \mathfrak{m}^{*}\right\rangle$. We conclude that $\left\{\left(M^{*}\right)_{0}: M \in \mathscr{N}\right\}$ is cofinal in $\left\{M_{0}: M \in \mathscr{N}\right\}$.

The next Lemma is the crucial final step.

LEMMA 4.6. Assume that $f: G \rightarrow H$ is a bijective morphism between connected pro-Lie groups. Then $f$ is open, that is, $f$ is an isomorphism.

PROOF. In the following we write $f^{-1}(N)_{0}$ as a hardly ambiguous abbreviation for $\left(f^{-1}(N)\right)_{0}$. We know that $\left\{\left(M^{*}\right)_{0}: M \in \mathscr{N}(G)\right\}=\left\{f^{-1}(N)_{0}: N \in \mathscr{N}(H)\right\}$ is cofinal in $\left\{P_{0}: P \in \mathscr{N}(G)\right\}$ and so

$$
\gamma: G \rightarrow \lim _{N \in \mathcal{N}(H)} G / f^{-1}(N)_{0} \quad \text { given by } \quad \gamma(g)=\left(g f^{-1}(N)_{0}\right)_{N \in \mathscr{N}(H)}
$$

is an isomorphism. Now $f\left(f^{-1}(N)_{0}\right)$ is connected and contained in $f\left(f^{-1}(N)\right)=N$ and thus $f\left(f^{-1}(N)_{0}\right) \subseteq N_{0}$. For $\mathfrak{n}=\mathscr{L}(N)$ and $\mathfrak{m}=\mathscr{L}(f)^{-1}(\mathfrak{n})$, because 
of $f \circ \exp _{G}=\exp _{H}$ and by Theorem 2.3(ii), we have $N_{0}=\overline{\left\langle\exp _{H} \mathfrak{n}\right\rangle}$ and $\exp _{G} \mathrm{~m} \subseteq$ $f^{-1}(N)_{0}$, whence $\exp _{H} \mathfrak{n} \subseteq f\left(f^{-1}(N)_{0}\right)$. Therefore $N_{0} \subseteq f\left(f^{-1}(N)_{0}\right)$. Hence $N_{0}=f\left(f^{-1}(N)_{0}\right)$ for all $N \in \mathscr{N}(H)$.

Since $f$ is bijective, for every $N \in \mathscr{N}(H)$ we have

$$
f^{-1}\left(N_{0}\right)=f^{-1}(N)_{0}
$$

Thus we have, for every $N \in \mathscr{N}(H)$, a bijective morphism

$$
\alpha_{N}: G / f^{-1}(N)_{0} \rightarrow H / N_{0} \text { given by } \alpha_{N}\left(g f^{-1}(N)_{0}\right)=f(g) N_{0} .
$$

Since $f^{-1}(N) \in \mathscr{N}(G)$ by Lemma 4.4, the factor group $G / f^{-1}(N)_{0}$ is locally compact by Proposition 2.10(vii), as is the factor group $H / N_{0}$. Since the group $G$ is connected, $G / f^{-1}(N)_{0}$ is $\sigma$-compact. The Open Mapping Theorem (Theorem A) applies and shows that $\alpha_{N}$ is an isomorphism for each $N \in \mathscr{N}(H)$. This gives us a morphism

$$
\begin{aligned}
\alpha: \lim _{N \in \mathcal{N}(H)} G / f^{-1}(N)_{0} & \rightarrow \lim _{N \in \mathcal{N}(H)} H / N_{0}, \\
\left(g_{N} f^{-1}(N)_{0}\right)_{N \in \mathcal{N}(H)} & \mapsto\left(f\left(g_{N}\right) N_{0}\right)_{N \in \mathscr{N}(H)}
\end{aligned}
$$

which is represented in the following diagram

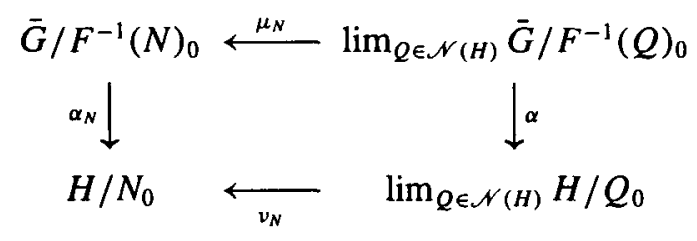

for the respective limit morphisms $\mu, \nu$.

Since $\gamma: G \rightarrow \lim _{N \in \mathcal{N}(H)} G / f^{-1}(N)_{0}$ given by $\gamma(g)=\left(g f^{-1}(N)_{0}\right)_{N \in \mathscr{N}(H)}$ is an isomorphism by (4.1), and by Proposition 2.10(iii), $\gamma_{\mathcal{N}_{0}(H)}: H \rightarrow \lim _{N \in \mathcal{N}(H)} H / N_{0}$ where $\mathscr{N}_{0}(H)=\left\{N_{0}: N \in \mathscr{N}(H)\right\}$ is an isomorphism, we may write

$$
f=\gamma_{\mathcal{N}_{0}(H)}^{-1} \circ \alpha \circ \gamma: \bar{G} \rightarrow H .
$$

Since $f$ is bijective, $\alpha$ is bijective. In order to prove that $f$ is open, it now suffices to show that $\alpha$ is an open morphism.

Each neighborhood of the identity in $\lim _{Q \in \mathcal{N}(H)} G / f^{-1}(Q)_{0}$ contains a neighbourhood of the identity of the form $W \stackrel{\text { def }}{=} \mu_{N}^{-1}(V)$ for a suitable $N \in \mathscr{N}(H)$ and some open neighborhood $V$ of the identity in $G / f^{-1}(N)_{0}$. Then by the openness of $\alpha_{N}$ the set $U \stackrel{\text { def }}{=} \alpha_{N}(V)$ is an open neighborhood of the identity in $H / N_{0}$. We claim that $\alpha(W)=\nu_{N}^{-1}(U)$. By the continuity of $\nu_{N}$, this claim will show that $\alpha(W)$ is open, and this will show the openness of $\alpha$. For a proof of the claim, 
we note that the surjectivity of $\mu_{N}$ and the commutativity of the diagram implies $U=\alpha_{N}(V)=\alpha_{N}\left(\mu_{N}(W)\right)=v_{N}(\alpha(W))$, whence $\alpha(W) \subseteq v_{N}^{-1}(U)$. For the proof of the converse inclusion, let $x \in \nu_{N}^{-1}(U)$. Then $\nu_{N}(x) \in U$ and since $\alpha_{N}$ is bijective, there is a $v \in V$ such that $\alpha_{N}(v)=\nu_{N}(x)$. By the definition of $W$, there is a $w \in W$ such that $\mu_{N}(w)=v$. Then $\nu_{N}(\alpha(w))=\alpha_{N}\left(\mu_{N}(w)\right)=u=v_{N}(x)$. Therefore $x^{-1} \alpha(w) \in \operatorname{ker} \nu_{N}$ for $N \in \mathscr{N}(H)$. But

$$
\begin{aligned}
W & =\mu_{N}^{-1}(V)=\mu_{N}^{-1} \alpha_{N}^{-1}(U)=\left(\alpha_{N} \mu_{N}\right)^{-1}(U) \supseteq\left(\alpha_{N} \mu_{N}\right)^{-1}(1)=\left(v_{N} \alpha\right)^{-1}(1) \\
& =\alpha^{-1}\left(\operatorname{ker} v_{N}\right)
\end{aligned}
$$

and, since $\alpha$ is surjective, there is a $w^{\prime} \in W$ such that $\alpha\left(w^{\prime}\right)=x^{-1} \alpha(w)$. Hence $x=\alpha\left(w w^{\prime-1}\right) \in \alpha(W)$. Thus the claim $\alpha(W)=v^{-1}(U)$ is established and this proves that $\alpha$ is open.

In the process we have reproved that the limit of an inverse system of isomorphisms is an isomorphism.

Lemma 4.6 proves that any bijective morphism $f: G \rightarrow H$ between connected pro-Lie groups is an isomorphism. Thus Lemma 4.3, and thereby the Open Mapping Theorem for Pro-Lie Groups (Theorem D), is proved.

\section{Consequences of the Open Mapping Theorem}

We remarked that a quotient of a pro-Lie group need not be a pro-Lie group. In Theorem 2.6 we saw circumstances in which the quotient of an almost connected pro-Lie group is a pro-Lie group. The following is another instance where a quotient is well behaved:

COROLlaRY 5.1. Let $G$ be an almost connected pro-Lie group and $N$ a normal subgroup which is the kernel of a morphism from $G$ onto a pro-Lie group. Then $G / N$ is a pro-Lie group.

PROOF. The preceding theorem applies to a morphism $f: G \rightarrow H$ from $G$ onto a pro-Lie group $H$ with $N=\operatorname{ker} f$. The induced bijective morphism $f^{\prime}: G / N \rightarrow H$ is an isomorphism and therefore $G / N$ is a pro-Lie group since $H$ is a pro-Lie group.

With an Open Mapping Theorem there is normally associated what is called a Closed Graph Theorem.

CoRollary 5.2 (Closed Graph Theorem for Pro-Lie Groups). Assume that $G$ and $H$ are pro-Lie groups, and that $f: G \rightarrow H$ is a morphism of groups (algebraically). Then $f$ is continuous if the graph $\operatorname{graph}(f) \stackrel{\text { def }}{=}\{(x, f(x)): x \in G\} \subseteq G \times H$ is closed in $G \times H$ and is almost connected. 
Proof. We define $\gamma: G \rightarrow \operatorname{graph}(f)$ by $\gamma(x)=(x, f(x))$ and decompose $f$ as follows:

$$
G \stackrel{\gamma}{\longrightarrow} \operatorname{graph}(f) \stackrel{\mathrm{pr}_{H} \operatorname{lgraph}_{(f)}}{\longrightarrow} H
$$

We see that $f$ is continuous if $\gamma$ is continuous. The continuity of $\gamma$ is equivalent to the openness of $\gamma^{-1}=\left.\operatorname{pr}_{G}\right|_{\operatorname{graph}(f)}$. By (ii), graph $(f)$ is a closed subgroup of the pro-Lie group $G \times H$. By the Closed Subgroup Theorem of pro-Lie groups (see [9, Theorem 3.35]), closed subgroups of pro-Lie groups are pro-Lie groups. Now the Open Mapping Theorem for Pro-Lie Groups applies to the continuous morphism

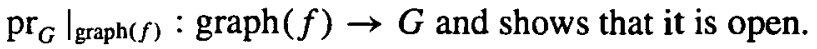

Of course the graph of any continuous function from a topological space to a Hausdorff space is closed.

If $H$ is a closed subgroup and $N$ a closed normal subgroup of a topological group $G$, then there is a natural bijective continuous morphism $\beta: H /(H \cap N) \rightarrow G / N$ given by $\beta(h(H \cap N))=h N$. Whenever $\beta$ is an isomorphism of topological groups one refers to this statement as the Second Isomorphism Theorem of Group Theory. Unfortunately, in general, the Second Isomorphism Theorem is not guaranteed for topological groups without additional hypotheses. For pro-Lie groups, due to the Open Mapping Theorem, the situation is much better. So let us assume that $H$ and $N$ are both almost connected. Then the semidirect product $N \rtimes_{l} H$ for $\iota(h)(n)=h n h^{-1}$ is almost connected and we have a surjective morphism $\mu: N \rtimes_{\imath} H \rightarrow N H$ given by $f(n, h)=n h$ with kernel $\left\{\left(h^{-1}, h\right): h \in H \cap N\right\} \cong H \cap N$. If now $N H$ is assumed to be a pro-Lie group, which is certainly the case if $G$ is a pro-Lie group and $H N$ is closed in $G$, by the Closed Subgroup Theorem ([9, Theorem 3.35]), then the Open Mapping Theorem for pro-Lie Groups shows that $\mu$ is open, giving us an exact sequence

$$
1 \rightarrow H \cap N \stackrel{h \mapsto\left(h^{-1}, h\right)}{\longrightarrow} N \rtimes_{1} H \stackrel{\mu}{\longrightarrow} N H \rightarrow 1
$$

(Mayer-Vietoris). The quotient map $q: H N \rightarrow H N / N$ is open and there is a commutative diagram

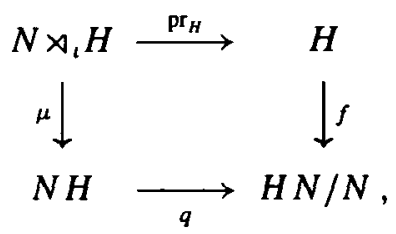

where $f(h)=h N$. It follows that $f$ is open. (This also follows from a direct application of the Quotient Theorem to $H N / N$ and the Open Mapping Theorem to $f$ 
plus the remark that continuous homomorphic images of almost connected groups are almost connected.) Since $\operatorname{ker} f=H \cap N$, the bijective morphism

$$
\beta: H /(H \cap N) \rightarrow H N / N
$$

induced by $f$ is an isomorphism. Thus, recalling that a complete subgroup of a topological group is closed, we have the following result:

COROLlaRY 5.3 (The Second Isomorphism Theorem for Pro-Lie groups). Assume that $H$ and $N$ are almost connected subgroups of a topological group, and that $N$ is normal. Assume further that that the group $H$, the group $N$, and their product $N H=H N$ are pro-Lie groups. Then the natural morphism

$$
\begin{gathered}
H /(H \cap N) \rightarrow H N / N \\
h(H \cap N) \mapsto h N
\end{gathered}
$$

is an isomorphism of topological groups.

In [9, Theorem 12.88] the following consequence of the Open Mapping Theorem for Pro-Lie Groups is established:

COROLlary 5.4 (An Alternative Open Mapping Theorem for Pro-Lie Groups). Let $f: G \rightarrow H$ be a surjective morphism between pro-Lie groups and assume that

(i) $G / \operatorname{ker} f$ is a pro-Lie group and

(ii) $H$ is connected and

(iii) $\mathscr{L}(f): \mathscr{L}(G) \rightarrow \mathscr{L}(H)$ is surjective.

Then $f$ is open.

\section{Acknowledgements}

We gratefully acknowledge correspondence with A. Adel George Michael through which we were alerted to a gap in an earlier version of our argument.

We also wish to express our appreciation to copy editor Jason Whyte for his assistance in the preparation of our text.

\section{References}

[1] N. Bourbaki, Groupes et algèbres de Lie, chapters 2-3 (Hermann, Paris, 1972).

[2] —, Topologie générale, chapters 5-10 (Hermann, Paris, 1974). 
[3] E. Hewitt and K. A. Ross, Abstract Harmonic Analysis. Vol. I Structure of topological groups. Integration theory, group representations (Academic Press, Inc., Publishers, New York, 1963).

[4] K. H. Hofmann, 'On a category of topological groups suitable for a structure theory of locally compact groups', Topology Proceedings 26 (2001-2002), 651-665.

[5] K. H. Hofmann and S. A. Morris, The Structure of Compact Groups (De Gruyter Berlin, 1998).

[6] - 'Projective limits of finite dimensional Lie groups', Proc. London Math. Soc. 87 (2003), 647-676.

[7] —_ 'The structure of abelian pro-Lie groups', Math. Z. 248 (2004), 867-891.

[8] - 'Sophus Lie's third fundamental theorem and the adjoint functor theorem', J. Group Theory 8 (2005), 115-133.

[9] - The Lie Theory of Connected Pro-Lie Groups- the Structure of Pro-Lie Algebra, Pro-Lie Groups and Locally Compact Groups (EMS Publishing House, Zürich, 2007).

[10] K. H. Hofmann, S. A. Morris and D. Poguntke, 'The exponential function of locally connected compact abelian groups', Forum Math. 16 (2003), 1-16.

[11] F. Burton Jones, 'Connected and disconnected plane sets and the functional equation $f(x+y)=$ $f(x)+f(y)$ ', Bull. Amer. Math. Soc. 48 (1942), 115-120.

[12] Sh. Koshi and M. Takesaki, 'An open mapping theorem on homogeneous spaces', J. Aust. Math. Soc., Ser. A. 53 (1992), 51-54.

[13] D. Montgomery and L. Zippin, Topological Transformation Groups (Interscience Publishers, New York, 1955).

[14] W. Roelcke and S. Dierolf, Uniform Structures on Topological Groups and their Quotients (McGraw-Hill, New York, 1981).

[15] H. Yamabe, 'Generalization of a theorem of Gleason', Ann. of Math. 58 (1953), 351-365.

[16] _ 'On the conjecture of Iwasawa and Gleason', Ann. of Math. 58 (1953), 48-54.

Fachbereich Mathematik

Darmstadt University of Technology

Schlossgartenstr. 7

D-64289 Darmstadt

Germany

e-mail: hofmann@mathematik.tu-darmstadt.de
School of Information Technology and Mathematical Sciences

University of Ballarat P.O. Box 663

Ballarat Victoria 3353

Australia

e-mail: s.morris@ballarat.edu.au 
J. Aust. Math. Soc. 83 (2007) 